\title{
Failure Recovery Dependency Analysis for Web Composition Transactions Based on Extended Petri-Nets
}

\author{
Mei Xiaoyong ${ }^{1,2}$, Huang Changqin ${ }^{1,3}$, Tang Yong $^{3}$, Zhao Gansen ${ }^{3}$ and Wang \\ Huijin $^{3}$ \\ 1. College of Educational Information and Technology, South China Normal \\ University, Guangzhou 510631, China \\ 2. School of Computer Science and Technology, Hunan University of Arts and \\ Science, Changde 415000, China \\ 3. Research Center for Information Services and Software Technology, South \\ China Normal University, Guangzhou 510631, China \\ cdmxy@126.com
}

\begin{abstract}
For transactional Composition Web Services (CWS), traditional transaction theory in the database is introduced into the Long Running Transactions (LRTs) domain. In practical application, the typical LRTs employ forward or backward rollback. In order to improve the expansibility of LRTs, it is important to accurately specify the dependency between tasks in composition transaction; contemporary technologies usually statically specify dependency point and avoid implicit interaction in LRTs. In this paper, we propose a task dependency modeling method for LRTs, firstly we present formal description of atomic Web Services (WS) and discuss atomic WS such as pivot, compensable, retriable and vital, then execution logs of LRTs data dependency and action dependency rules between tasks in LRTs are analyzed, specify, finally application of TRP shows that it is feasible to ensure consistent execution of reliable LRTs based dependencies.
\end{abstract}

Keywords: Transactional Composition Web Services, Task Dependency, Compensation, Execution log

\section{Introduction}

CWS composed a set of heterogeneous, autonomous and loosely coupled WS to offer new value-added services. Since the long-lived nature of CWS, it brings difficulty to transaction handling. Therefore, it needs to improve the failure handling capability to tolerate the occurrence of failure and finally coordinate it to a consistent acceptable state. In a loosely coupled LRTs environment, it is inevitable to take more relax transaction mechanism, which is called relaxed-ACID.

When using LRTs to composite WS, LRTs define compensation strategies and enclosed tasks in LRT will be compensated one by one from the innermost level until it terminates successfully. N. B. Lakhal and J. E. Haddad et al. discuss task dependency between nested layers in composition transaction, the failure in lower layer may propagate to a higher layer [1-2]. Since there are data dependency between composition transactions, failure of a task may lead to the inconsistency of other tasks. T.W. Chen and J. Miao discuss execution dependencies and compensation dependencies among tasks in business processes [3]. R.T. Khachana, A. Jamesa et al. introduce a new model for supporting transactions in a web environment [4]. More advanced data dependency approaches rely on comparing structured data such as parameters types and names [5]. This is generally not enough to identify the dependencies between tasks in terms of 
functionality, and consequently makes it difficult, or even impossible, to use these methods to automate LRTs processing. L. Garc $1 \mathrm{a}$-Banuelos proposes a executable transaction model based on ASML that allows seamless add/modify behavior, and extends failure handler in WS-BPEL [6-7]. R. Hamadi and B. Benatallan propose a SelfAdaptation Recovery Net (SARN) based on extended Petri nets model to specify fault or exceptional behavior in business process [7-8]. Due to the complexity of these kinds of transaction process, multiple dependencies such as temporal dependency, data flow dependency, control flow dependency, state dependency and behavior dependency often need to be constructed simultaneously to execute the transaction process in composition transaction environments. Hence the dependency problem between tasks in LRTs must be considered, which affects the effectiveness and efficiency of failure compensation of composition transaction.

\section{Composition Transaction Dependency}

How to ensure reliable and accurate execution of composition transaction is a important problem in transaction process. Based on the analysis of transaction execution logs, transaction dependency rules are constructed and misbehaviors that may cause unexpected results are detected, which handle failure, and ensure reliable and consistent execution of transaction.

\subsection{Data Flow Dependency}

The dependency of Web transaction relies on the control flow and the data flow of aggregation pattern. During the execution of composition business process, the data flow always follows the control flow. The explicit and implicit interaction in a data flow may violate atomicity of LRTs, explicit interaction in LRTs usually means the output of the previous task is the input of the current task and there is obvious partial order between two tasks, dependency rules are described as follows:

\section{Rule 1}

(before $\left(I_{j}, I_{i}\right) \vee \operatorname{contain}\left(I_{j}, I_{i}\right) \vee$ meets $\left.\left(I_{j}, I_{i}\right) \vee \operatorname{starts}\left(I_{j}, I_{i}\right)\right) \wedge I_{i}$.precond $\left(I_{j}\right.$.Activate ()$) \wedge\left(I_{j}\right.$.out $\bigcap$ $I_{i}$ in $\left.\neq \varnothing\right) \Rightarrow I_{i} \stackrel{\text { exd }}{\longrightarrow} I_{j}$.

Task $I_{i}$ explicitly depends on $I_{j}$.

Rule $2 I_{i} \stackrel{\text { exd }}{\longrightarrow} I_{k}, I_{k} \stackrel{\text { exd }}{\longrightarrow} I_{j} \Rightarrow I_{i} \stackrel{\text { inexd }}{\longrightarrow} I_{k}$.

Task $I_{i}$ explicitly depends on $I_{j}$ indirectly. More generally, $I_{i} \stackrel{\text { exd }}{\longrightarrow} I_{1}, I_{1} \stackrel{\text { exd }}{\longrightarrow} I_{2}, \cdots$, $I_{k} \stackrel{\text { exd }}{\longrightarrow} I_{j} \Rightarrow I_{i} \stackrel{\text { inexd }}{\longrightarrow} I_{j}$, where $\sigma=I_{1} I_{2} \cdots I_{k}(k>1)$.

Besides the mentioned explicit data dependency, there is implicit data interaction dependency between tasks of LRTs. For simplify analysis, def-use relationship [9-10] is introduced to locate this implicit data interaction. def-use $\left(I_{i}, I_{j}\right)$ means, for two given tasks $I_{i}$ and $I_{j}, I_{i}$ is committed before $I_{j}$ and $I_{j}$ should use the data defined by $I_{i}$. The relaxed def-use' $\left(I_{i}, I_{j}\right)$ means, for two given tasks $I_{i}, I_{j}, I_{i}$ is committed before or together with $I_{j}$, and $I_{j}$ should use the data defined by $I_{i}$.

Rule 3

(equals $\left(I_{i}, I_{j}\right) \vee \operatorname{contain}\left(I_{i}, I_{j}\right) \vee \operatorname{starts}\left(I_{i}, I_{j}\right) \vee$ finishes $\left.\left(I_{i}, I_{j}\right)\right) \wedge$ def-use $\left(t_{i}, t_{j}\right) \Rightarrow I_{i} \stackrel{\text { imd }}{\longrightarrow} I_{j}$.

Task $I_{i}$ implicitly depends on $I_{j}$.

Rule $4 I_{i} \stackrel{i m d}{\longrightarrow} I_{k}, I_{k} \stackrel{i m d}{\longrightarrow} I_{j} \Rightarrow I_{i} \stackrel{i m d}{I_{k}} I_{j}$.

Task $I_{j}$ implicitly depends on $I_{i}$ indirectly. More

generally, $I_{i} \stackrel{\text { imd }}{\longrightarrow} I_{1}, I_{1} \stackrel{\text { ind }}{\longrightarrow} I_{2}, \cdots, I_{k} \stackrel{\text { imd }}{\longrightarrow} I_{j} \Rightarrow I_{i} \stackrel{\text { inimd }}{\longrightarrow} I_{j}$, where $\sigma=I_{1} I_{2} \cdots I_{k}(k>1)$. 


\subsection{Action Dependency}

Rule $5 I_{i}$.commit ()$\wedge\left(\right.$ before $\left(I_{i}, I_{j}\right) \vee$ meets $\left.\left(I_{i}, I_{j}\right)\right) \rightarrow I_{j}$.Activate ()$\Rightarrow I_{j} \stackrel{\text { Act }}{\longrightarrow} I_{i}$.

Rule $6\left(I_{i}\right.$.Abort ()$\vee I_{i}$.Fail ()$\vee I_{i}$.Cancel ()$) \wedge\left(\left(\right.\right.$ before $\left(I_{i}, I_{j}\right) \vee$ meets $\left.\left(I_{i}, I_{j}\right)\right) \wedge I_{j}$.Activated $\rightarrow$ $I_{j}$. Abort ()$\Rightarrow I_{j} \stackrel{A b t}{\longrightarrow} I_{i}$.

Rule $7\left(I_{i}\right.$.Fail ()$\vee I_{i}$.Cancel ()$) \wedge\left(\right.$ finishes $\left(I_{i}, I_{j}\right) \vee$ equals $\left.\left(I_{i}, I_{j}\right)\right) \wedge I_{j}$.Running $\rightarrow I_{j}$.Abort ()$\Rightarrow$ $I_{j} \stackrel{\mathrm{Cnl}}{\longrightarrow} I_{i}$.

Rule $8 I_{i} \cdot$ Fail ()$\wedge\left(I_{i} \equiv I_{i}^{\prime \prime}\right) \rightarrow I_{i}^{\prime \prime}$.Activate ()$\Rightarrow I_{i}^{\prime \prime} \stackrel{\text { Alt }}{\longrightarrow} I_{i}$.

$I_{j}^{\prime \prime} \stackrel{A l t}{\longrightarrow} I_{j}$ or $I_{k}^{\prime \prime} \stackrel{A t t}{\longrightarrow} I_{k}$ is satisfied in AND-join; $I_{k} \stackrel{\text { Alt }}{\longrightarrow} I_{k}^{\prime \prime}$ is satisfied in AND-split .

According to the semantics of $\stackrel{A b t}{\longrightarrow}$ and $\stackrel{C n l}{\longrightarrow}, \stackrel{A b t}{\longrightarrow}$ should abort the tasks that haven't started and $\stackrel{\mathrm{Cnl}}{\longrightarrow}$ should cancel the tasks under execution. Based on Rule 5Rule 8, composition rules can be deduced as follows:

Rule $9 I_{i} \stackrel{C m t}{\longrightarrow} I_{j}, I_{j} \stackrel{C m t}{\longrightarrow} I_{k} \Rightarrow I_{i} \stackrel{C m t}{\longrightarrow} I_{k}$;

Rule $10 I_{i} \stackrel{C m t}{\longrightarrow} I_{j}, I_{i} \stackrel{C m t}{\longrightarrow} I_{k} \Rightarrow I_{i} \stackrel{C m t}{\longrightarrow}\left(I_{j} \wedge I_{k}\right)$;

Rule $11 I_{i} \stackrel{\mathrm{Cmt}}{\longrightarrow} I_{k}, I_{j} \stackrel{\mathrm{Cmt}}{\longrightarrow} I_{k} \Rightarrow\left(I_{i} \vee I_{j}\right) \stackrel{\mathrm{Cmt}}{\longrightarrow} I_{k}$

Rule $12 I_{i} \stackrel{\mathrm{Cmt}}{\longrightarrow} I_{j}, I_{j} \stackrel{\mathrm{Act}}{\longrightarrow} I_{k} \Rightarrow I_{i} \stackrel{A c t}{\longrightarrow} I_{k}$;

Rule $13 I_{i} \stackrel{A c t}{\longrightarrow} I_{j}, I_{i} \stackrel{A c t}{\longrightarrow} I_{k} \Rightarrow I_{i} \stackrel{A c t}{\longrightarrow}\left(I_{j} \wedge I_{k}\right)$;

Rule $14 I_{i} \stackrel{C m t}{\longrightarrow} I_{j},\left(I_{i} \vee I_{j}\right) \stackrel{A c t}{\longrightarrow} I_{k} \Rightarrow\left(I_{i} \wedge I_{j}\right) \stackrel{A c t}{\longrightarrow} I_{k}$

Rule $15 I_{i} \stackrel{C m t}{\longrightarrow} I_{j},\left(I_{j} \wedge I_{k}\right) \stackrel{A b t}{\longrightarrow} I_{s} \Rightarrow\left(I_{i} \wedge I_{k}\right) \stackrel{A b t}{\longrightarrow} I_{s}$

Rule $16 I_{i} \stackrel{C m t}{\longrightarrow} I_{j},\left(I_{j} \vee I_{k}\right) \stackrel{A b t}{\longrightarrow} I_{s} \Rightarrow\left(I_{i} \vee I_{k}\right) \stackrel{A b t}{\longrightarrow} I_{s}$

Rule $17 I_{i} \stackrel{\mathrm{Cnl}}{\longrightarrow} I_{j}, I_{j} \stackrel{\mathrm{Cnl}}{\longrightarrow} I_{k} \Rightarrow I_{i} \stackrel{\mathrm{Cnl}}{\longrightarrow} I_{k}$;

Rule $18 I_{i} \stackrel{C n l}{\longrightarrow} I_{j} \Rightarrow\left(I_{i}\left\|I_{1}\right\| I_{2}\|\cdot \cdot\| I_{m}\right) \stackrel{C n l}{\longrightarrow} I_{j}$;

If $I_{j}$ sends $I_{j}$.Fail () or $I_{j}$.Compensated () to $I_{i}$, then there exist the following compensation rules between $I_{i}$ and $I_{j}$ :

Rule 19

$I_{j} \stackrel{\text { exd }}{\longrightarrow} I_{i} \wedge\left(I_{i} . T B P \in\{\right.$ Compensable, Vital $\left.\}\right) \wedge I_{i}$.Committed $\wedge\left(I_{j}\right.$. Fail ()$\vee I_{j}$. Compensate ()$)$

$\rightarrow I_{i}$.Compensate ()$\Rightarrow I_{i} \stackrel{C_{p t e d}}{\longrightarrow} I_{j}$;

Rule 20

$\left(I_{j} \stackrel{\text { inexd }}{\omega^{\prime}} \rightarrow I_{i} \vee I_{j} \stackrel{\text { inexd }}{\longrightarrow} I_{i}\right) \wedge(I . T B P \in\{$ Vital, Compensable $\}) \wedge I_{i}$.Committed $\wedge\left(I_{j}\right.$. Fail ()$\vee$

$I_{j}$.Compensate ()$) \rightarrow I_{i}$.Compensate ()$\Rightarrow I_{i} \stackrel{C_{p t i n e x d}}{\longrightarrow} I_{j}$;

\section{Rule 21}

$\left(I_{j} \stackrel{\text { inexd }}{\omega^{\prime}} \rightarrow I_{i} \vee I_{j} \stackrel{\text { inexd }}{\longrightarrow} I_{k}\right) \wedge(I . T B P \in\{$ Vital, Compensable $\}) \wedge I_{i}$. Committed $\wedge\left(I_{j}\right.$. Fail ()

$\vee I_{j}$.Compensate ()$) \rightarrow I_{i}$.Compensate ()$\Rightarrow I_{i} \stackrel{\text { Cptind }}{\longrightarrow} I_{j}$;

Rule 22

$I_{j} \stackrel{\text { inimd }}{\longrightarrow} I_{i} \wedge I_{j} . T B P \in\{$ Compensable, Vital $\left.\}\right) \wedge I_{i}$.Committed $\wedge\left(I_{j}\right.$.Faile ()$\vee I_{j}$.Compensate ()$)$

$\rightarrow I_{i}$.Compensate ()$\Rightarrow I_{i} \stackrel{C p_{\text {tinum }}}{\longrightarrow} I_{j}$;

Aggregation control patterns are used in LRTs, such as $\oplus, \square \otimes, L$, where $\square$ is composed of And-Split and And-join, $\otimes$ is composed of Or-split and Or-join. Based on Rule 19Rule 22, composition rules can be deduced as follows:

Rule $23 I_{i} . T B P, I_{j} . T B P \in\{$ Compensable, Vital $\} \wedge I_{i} \oplus I_{j} \Rightarrow I_{j} \stackrel{C p t}{\longrightarrow} I_{i}$.

Rule $24 I_{i} \cdot T B P, I_{j} . T B P, I_{k} . T B P \in\{$ Compensable, Vital $\} \wedge I_{i} \oplus\left(I_{j} \square I_{k}\right) \Rightarrow\left(I_{j} \square I_{k}\right) \stackrel{C p t}{\longrightarrow} I_{i}$.

Rule $25 I_{i} \cdot T B P, I_{j} . T B P, I_{k} . T B P \in\{$ Compensable, Vital $\} \wedge\left(I_{i} \square I_{j}\right) \oplus I_{k} I_{k} \stackrel{C p t}{\longrightarrow}\left(I_{i} \square I_{j}\right)$.

Rule $26 I_{i} \cdot T B P, I_{j} . T B P, I_{k} . T B P \in\{$ Compensable, Vital $\} \wedge I_{i} \oplus\left(I_{j} \otimes I_{k}\right) \Rightarrow\left(I_{j} \otimes I_{k}\right) \stackrel{C p t}{\longrightarrow} I_{i}$.

Rule $27 I_{i} \cdot T B P, I_{j} . T B P, I_{k} . T B P \in\{$ Compensable, Vital $\} \wedge\left(I_{i} \otimes I_{j}\right) \oplus I_{k} \Rightarrow I_{k} \stackrel{C p t}{\longrightarrow}\left(I_{i} \otimes I_{j}\right)$. 
Rule $28 I_{i} \stackrel{C p t}{\longrightarrow} I_{j}, I_{j} \stackrel{C p t}{\longrightarrow} I_{k} \Rightarrow I_{i} \stackrel{C p t}{\longrightarrow} I_{k}$

Rule 29

$I_{j} \stackrel{C p t}{\longrightarrow} I_{k}, I_{k} \stackrel{C_{p t}}{\longrightarrow} I_{j}, I_{j} \stackrel{C n l}{\longrightarrow} I_{k}, I_{k} \stackrel{C n l}{\longrightarrow} I_{j}, I_{k} \stackrel{C_{p t}}{\longrightarrow}\left(I_{i} \square I_{j}\right) \Rightarrow\left(I_{j} \square I_{k}\right) \stackrel{C p t}{\longrightarrow} I_{i} ;$

Rule 30

$I_{i} \stackrel{C p t}{\longrightarrow} I_{j}, I_{j} \stackrel{C p t}{\longrightarrow} I_{i}, I_{i} \stackrel{C n l}{\longrightarrow} I_{j}, I_{j} \stackrel{C n l}{\longrightarrow} I_{i}, I_{i} \stackrel{A b t}{\longrightarrow} I_{j}, I_{j} \stackrel{A b t}{\longrightarrow} I_{i} \Rightarrow I_{k} \stackrel{C p t}{\longrightarrow}\left(I_{i} \square I_{j}\right) ;$

Rule 10-Rule 22 can be deduced from Rule 1- Rule 9. Consdiering the verification of flow structure of LRTs, if there exists AND-Join, but no AND-Split (or exists OR-Join, but no OR-Split), then there exist design defects in LRTs flow, deduced from Rule 9-Rule 30. Therefore, not all defects can be detected during execution according to rule deduction.

\section{Generation and Test Algorithm of Dependency Rules for LRTs}

For each task in LRTs, direct dependency between tasks is calculated and added to direct data dependency set DDs according to dependency rules. Then indirect data dependency set IDs is calculated between nonadjacent tasks according to DDs. Finally, DDs and IDs are merged together to calculate dependency set of LRTs. The generation dependency set algorithm is given as follows.

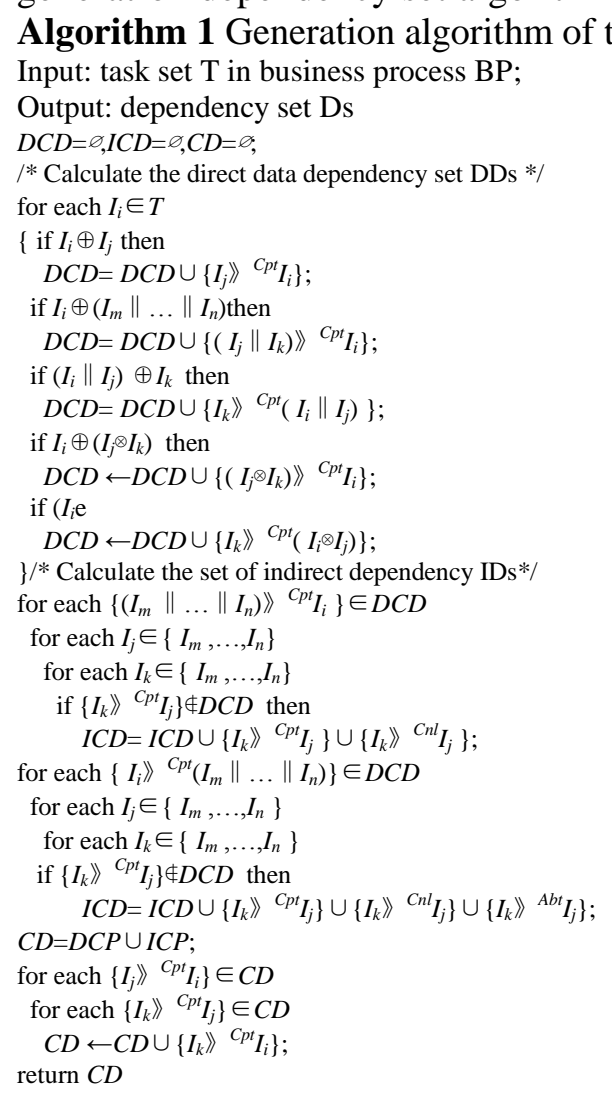

For given LRTs, if $|T|=n$, time complexity of generating compensation dependency set of adjacent tasks is $O\left(n^{2}\right)$, while for nonadjacent tasks it is $O\left(n^{3}\right)$, therefore, time complexity is $O\left(n^{3}\right)$.

DDG (Data Dependency Graph) and TDG (Task Dependency Graph) can be generated according to the exectuion logs and task dependencies in LRTs. For $\mathrm{x}, \mathrm{y} \in$ Logitem in DDG, there exist $I_{i}$ such that $x \stackrel{I_{i}}{\longrightarrow} y$; for $I_{i}, I_{j} \in \mathrm{TDG}$, there exist $\mathrm{x}$ such that $I_{i} \stackrel{x}{\longrightarrow} I_{j}$. 
Weighted graph is an intuitive way to represent the dependency degree between tasks, complete dependency and partial dependency between tasks are represented in Figure 1 and Figure 2 respectively, where node denotes task, edge denotes dependency, and weight denotes dependency degree. If there exists $I_{j} \rightarrow I_{i}\left(I_{j}^{\alpha} I_{i}\right)$, then dependency degree of $I_{i}$ and $I_{j}$ is $\alpha=(n-n e) / n$, where $n$ and $n e$ are the number of dependency instance and nondependency instance respectively. Then we can get a $n \times n$ TDM (Task Dependency Matrix), where each element $\alpha_{i j}$ denotes dependency degree of $I_{i}$ and $I_{j}$. For example, $I_{i} . o u t\left(\operatorname{par}_{1}, \operatorname{par}_{2}, \cdots\right.$, par $\left._{m}\right)$ and $I_{j}$. in $\left(\right.$ par $_{1}$, par $_{2}, \cdots$, par $\left._{k}\right)$, if $\mathrm{n}=\mathrm{m}+\mathrm{k}=11, \mathrm{ne}=3$, then $\alpha=(11-$ 3)/11 $=72.7 \%$, denoted as $I_{j}^{0.727} I_{i}$.

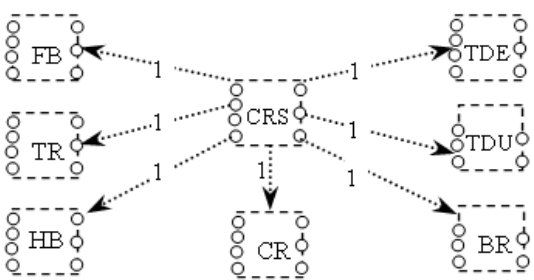

Figure 1. Complete Dependency

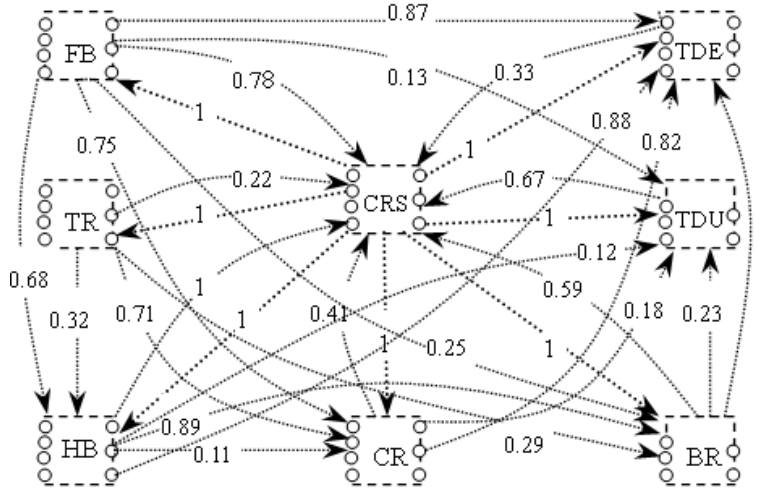

Figure 2. Partial Dependency

As shown in Figure 1 and Figure 2, it is obvious that partial dependency provide more information. For example, in Figure 2, there is a directed edge form FB to HB weighted 0.68 , we call $\mathrm{FB}$ is the dependency point of $\mathrm{HB}, \mathrm{HB}$ is the decision point of $\mathrm{FB}$, and 0.68 is the partial dependency degree.

Algorithm 2 Generation algorithm of DDG and TDG

Input: Logfile and Ds

Output: DDG and TDG

$\{$ For each data $\mathrm{x} \in \mathrm{Ds} / *$ datas that affectes transaction*/

For each datat $\mathrm{y} \in \mathrm{I}_{\mathrm{i}}$.data $/ *$ data item of transaction*/

[If $x \stackrel{I_{i}}{\longrightarrow} y \quad / *$ if there exist dependency*/

InsertDDG(x,y); /*insert to DDG*/

If $I_{i} \stackrel{x}{\longrightarrow} I_{j} / *$ if there exist dependency*/

InsertTDG $\left(\mathrm{I}_{\mathrm{i}}, \mathrm{I}_{\mathrm{i}}\right) ; / *$ insert to $\mathrm{TDG}^{*} /$

\})

It is very important to verify task dependency in LRTs. If all task dependencies in LRTs satisfy $\mathrm{TD}_{1}, \mathrm{TD}_{2}, \ldots, \mathrm{TD}_{\mathrm{n}}$, then $\mathrm{I}_{1} \rightarrow \mathrm{I}_{\mathrm{n}}$, that is, there exist at least one rule set in rule base such that LRTs execute from I1 to In. Henry et al. verify the logical expression correctness of this criterion with propositional logic [11]. This paper presents the verification algorithm of this criterion, which firstly verify basic dependency with Rule 5Rule 9; then verify composition dependency with Rule 10-Rule 30; finally if execution of $I_{1} \rightarrow I_{n}$ can be verified, then the execution of LRTs is correct.

Algorithm 3 Verify task dependency in LRTs.

Input: BP, TDG, DDG.

Output: Correctness of dependency.

Activated $=\{$ all the tasks whose state is Activated $\}$;

Committed $=\{$ all the tasks whose state is committed $\}$;

Aborted $=\{$ all the tasks whose state is aborted $\}$;

Cancelled $=\{$ all the tasks whose state is cancelled $\}$;

Compensated $=\{$ all the tasks whose state is compensated $\}$;

Failed $=\{$ all the tasks whose state is failed $\}$;

ExecutionTask $=\varnothing ;$ ActivationTask $=\varnothing ;$ CommitionTask $=\varnothing$;

AbortionTask $=\varnothing ;$ CancellationTask $=\varnothing$;

for each task $I i \in \mathrm{BP}\{$

for each task Ii.Activated \{ 


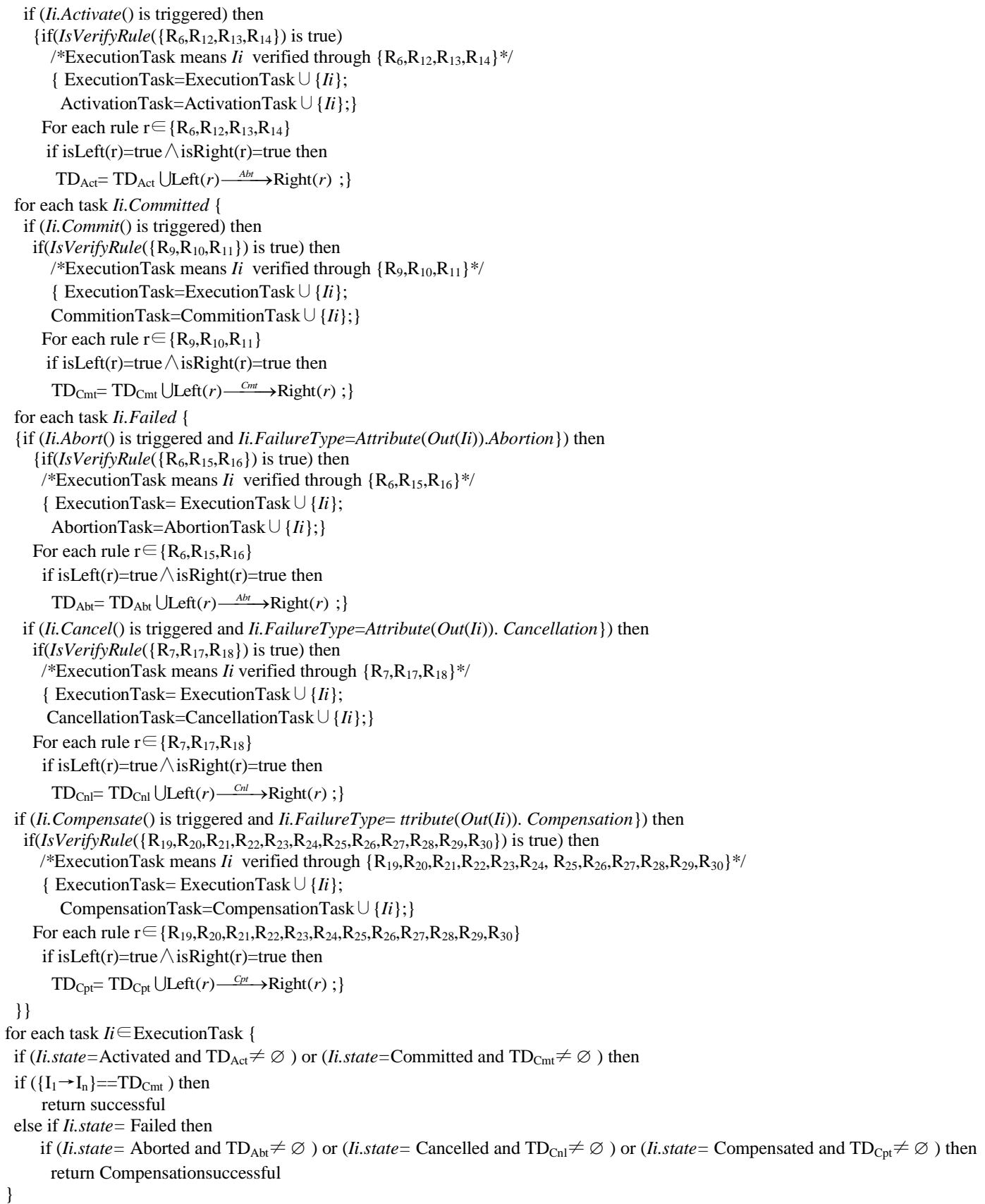

For given LRTs, if $|T|=n$ and $|\mathrm{TP}|=\mathrm{m}$, time complexity of verification algorithm is $O\left(n^{3}\right)$. If LRTs failed, dependency requirements of tasks are verified with $\mathrm{TD}_{\text {Act }}, \mathrm{TD}_{\mathrm{Cmt}}$, $\mathrm{TD}_{\mathrm{Abt}}, \mathrm{TD}_{\mathrm{Cnl}}, \mathrm{TD}_{\mathrm{Cpt}}$.

\section{Application of Composition Transaction}

We implement this case with the open source software ActiveBPEL [12], the experiment is implemented on a testbed consisting of 2 IBM x 3650 servers and 12 PCs connected by a 100Mbps Ethernet. Each server is equipped with 4 Intel $2930 \mathrm{MHz}$ processors and $1 \mathrm{~GB}$ memory running SuSE 10.2 Linux. Each PCs is equipped with Intel Pentium Dual Core E5200 2.5 GHz, 2GB RAM, Windows XP SP2. the process execution engine construct execution log to help reconstruct compensation context when binding compensation service, so reverse compensation flow is constructed, as shown in Fig. 3. $t_{1}^{f}, t_{2}^{f}, \cdots, t_{10}^{f}$ is introduced to construct the 
mapping between $I_{1}, I_{2}, \cdots, I_{10}$ and their corresponding compensation tasks $I_{1}^{\prime}, I_{2}^{\prime}, \cdots, I_{10}^{\prime}$. In practical applications, it needs to consider compensation strategy. During the execution of TRP, the failure of task will trigger $\mathrm{CH}$ to abort the forward flow and turn to the backward compensation flow. Based on the compensation pair, the $\begin{array}{lllll}\text { extended } & \text { TRP } & \text { is } & \text { as }\end{array}$ $\left.\left.\div F B^{\prime}\right) \otimes T R\right)\left\|\left(H B \div H B^{\prime}\right)\right\|\left(\left(C R \div C R^{\prime}\right) \otimes(B R \div\right.$

$\left.\left.B R^{\prime}\right)\right) \oplus\left(O P \div O P^{\prime}\right) \oplus\left(\left(T D E \div T D E^{\prime}\right) \otimes\left(T D U \div T D U^{\prime}\right)\right) \oplus T C$.

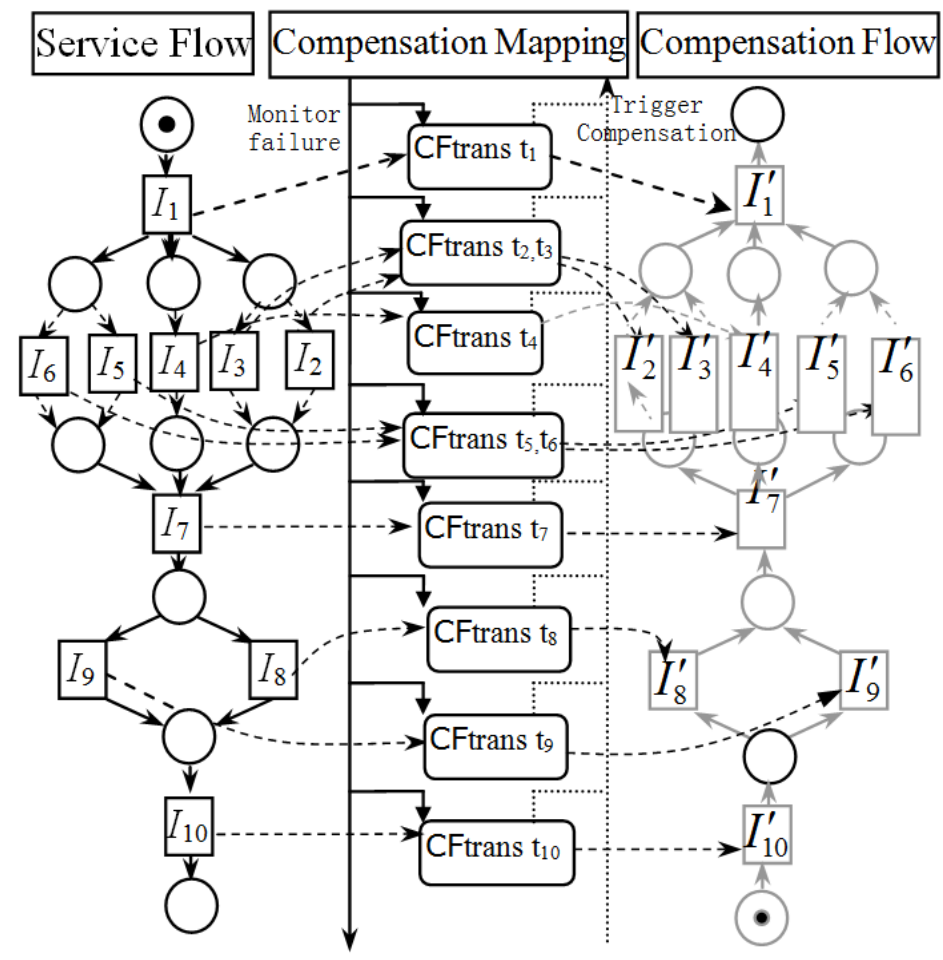

Figure 3. Multi-partner Cooperation Traveling Reservation Composition

To show the effectiveness of the proposed approach, we performed an experimental evaluation with a dependency- based WSC. Since the approach employed a generation algorithm of compensation dependency, it is feasible to execute it in a recovery environment. The result from the experiments that shows dependency rules based on recovery and the execution of TRP, as shown in Figure 4. The dependency-based recovery approach locates any conforming recovery service with the dependency rules and performs dependency reasoning. From these results, it is evident that the proposed approach is efficient and scalable than a nondependency-based approach which does not incorporate a facility to handle dependency. 


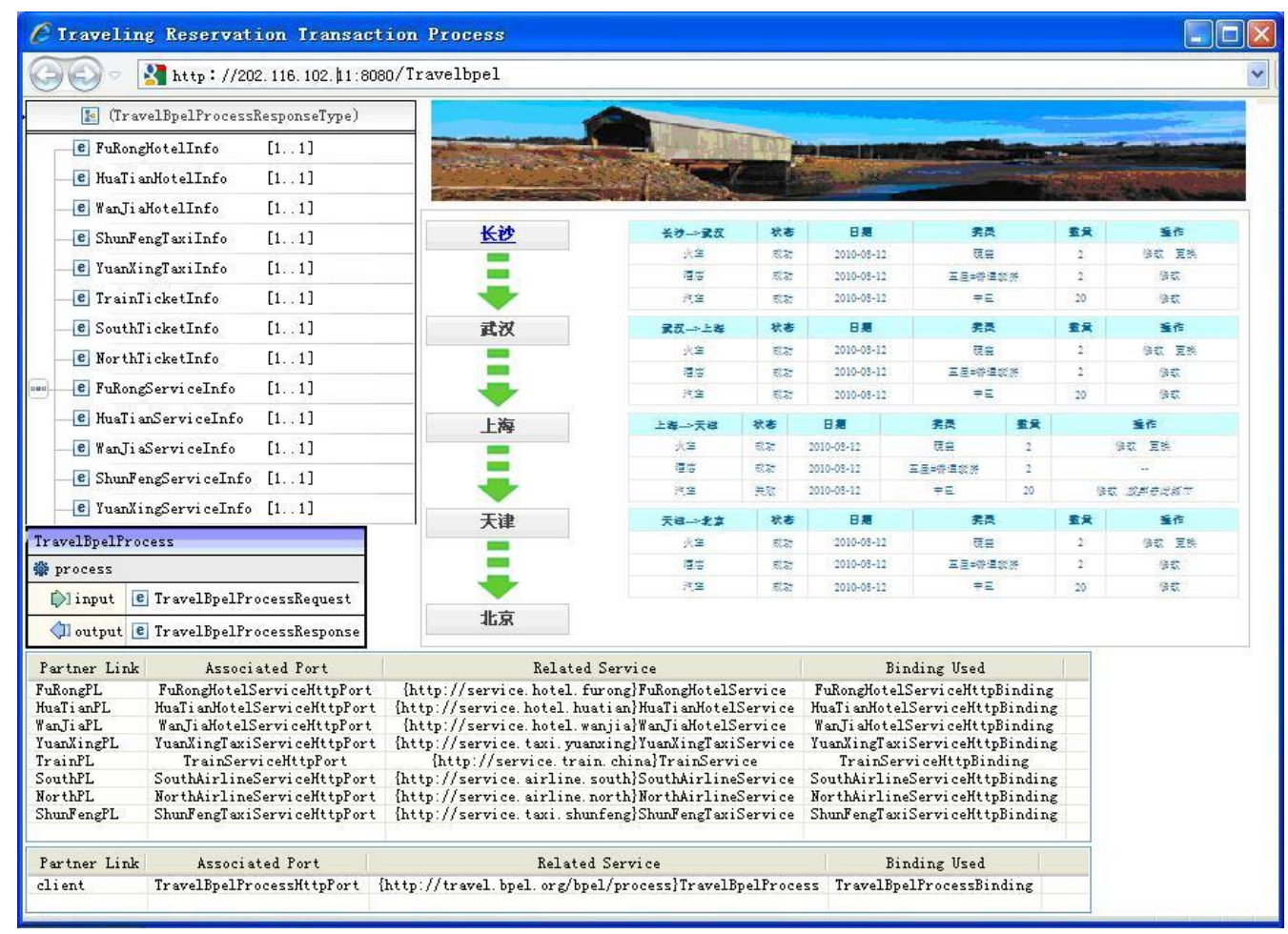

Figure 4. Execution Result of TRP

\section{Conclusion}

Current failure compensation strategies of composition transaction seldom take into consideration these dependencies. Therefore, in this paper, we proposes a task dependency method based on extended Petri nets using transaction execution log, firstly we formalize atomic WS based on Petrti nets and discusse atomic WS, then we analysis execution logs of LRTs, specify dataflow dependency and action dependency between tasks in LRTs, application of TRP based on LRTs shows that it is feasible to ensure reliable execution of LRTs and verify dependency of TRP.

\section{Acknowledgements}

This work is one of the projects supported by the National Key Technologies R\&D Program of China (2013BAH72B01), the National Natural Science Foundation of China (60940033, 61370229), the Postdoctoral Science Foundation of China (20080440121), the Natural Science Foundation of Province (06017089, 60940033), the Science and Technology Planning Project of Hunan Province (2010GK3020), Youth Foundation of Educational Commission of Hubei Province of China (12B092).

\section{References}

[1] N. B. Lakhal, T. Kobayashi and H. Yokota, "FENECIA: failure endurable nested-transaction based execution of composite Web services with incorporated state analysis", International Journal on Very Large Data Bases, vol.18, no.1, (2009), pp. 1-56.

[2] J. E. Haddad, M. Manouvrier and M. Rukoz, "TQoS: Transactional and QoS-Aware Selection Algorithm for Automatic Web Service Composition”, IEEE Transactions on Services Computing, vol. 3, no. 1, (2010), pp. 73-85.

[3] T. W. Chen and J. Miao, "Research on Exception Handling Mechanism Based on Directed Graph in Service Composition", Applied Mechanics and Materials, vol. 58, (2011), pp. 1088-1093. 
[4] R. T. Khachana, A. Jamesa and R. Iqbala, "Relaxation of ACID properties in AuTrA: The adaptive userdefined transaction relaxing approach", Future Generation Computer Systems, vol. 27, no. 1, (2011), pp. 58-66.

[5] J. Ma, Y. Zhang and J. He, "Web Services Discovery Based on Latent Semantic Approach", Proceedings of the 2008 IEEE International Conference on Web Services, (2008), pp. 740-747.

[6] L. Garcia-Banuelos, "An AsmL Executable Model for WS-BPEL with Orthogonal Transactional Behavior", 4th international conference Business process management, LNCS 4102, (2006), pp. 401406.

[7] A. Varga, A. E. C. Basaveb, M. Rowe, F. Ciravegna and Y. Hed, "Linked knowledge sources for topic classification of microposts: A semantic graph-based approach", Web Semantics: Science, Services and Agents on the World Wide Web, vol. 26, no. 4, (2014), pp. 36-57.

[8] R. Hamadi, B. Benatallah and B. Medjahed, "Self-adapting recovery nets for policy-driven exception handling in business processes", Distributed and Parallel Databases, vol. 23, no. 1, (2008), pp. 1-44.

[9] B. Medjahed and Z. Malik, "Bottom-Up Fault Management in Composite Web Services", Lecture Notes in Computer Science, vol. 6741, (2011), pp. 597-611.

[10] [9] A. Tahir, D. Tosi and S. Morasca, "A systematic review on the functional testing of semantic web services", Journal of Systems and Software, vol. 86, no. 11, (2013) November, pp. 2877-2889.

[11] F. E. Allen and J. Cocke, "A program data flow analysis procedure", Communications of the ACM, vol. 19, no. 3, (1976), pp. 137-146.

[12] H. H. Bi and J. L. Zhao, "Applying propositional logic to workflow verification", Information Technology and Management, vol. 5, no. 324, (2004), pp. 293 -318.

[13] Activebpel, http://www.activebpel.org/down_ load.

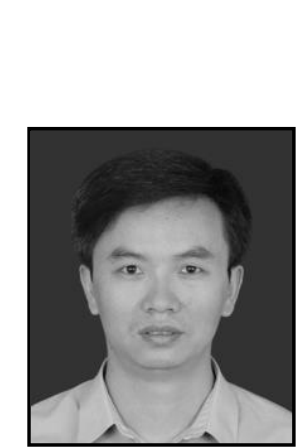

\section{Authors}

Mei Xiaoyong, he was born in 1974, Ph.D. professor. His research interests include service-oriented computing, semantic Web and Petri-nets.

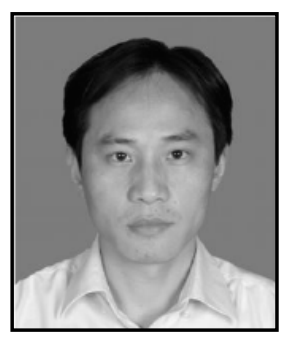

Huang Changqin, he was born in 1972, Ph.D. professor. His research interests include information technology and serviceoriented computing. 
International Journal of $u-$ and e- Service, Science and Technology Vol.8, No.4 (2015) 